\title{
Sentidos de aplicação e pontas de pulverização no percentual de cobertura em alvos artificiais, para diferentes situações de orientação de alvo e vento
}

\author{
Spray directions and nozzles in the percentage of coverage in artificial targets, to different situations \\ of target orientation and wind
}

\author{
Gustavo Migliorini de Oliveira' ${ }^{\mathrm{I}}$ Marcelo Gonçalves Balan II Inês Cristina de Batista Fonseca ${ }^{\mathrm{III}}$ \\ Otavio Jorge Grigoli Abi Saab ${ }^{\text {III }}$
}

\section{RESUMO}

\begin{abstract}
Diversos fatores influenciam na eficiência de uma aplicação via líquida: clima, equipamento, produto fitossanitário, tipo de alvo e momento biológico de controle. Outro ponto é a uniformidade da aplicação nas diferentes faces das folhas, pois influencia na absorção e translocação dos produtos sistêmicos e na ação de contato sobre o agente biológico que se quer controlar. $O$ objetivo do trabalho foi estudar o efeito de diferentes sentidos de aplicação e pontas de pulverização no percentual de cobertura em alvos artificiais (face superior e inferior) para seis diferentes situações de orientação de alvo e vento. Os alvos artificiais constituíram-se de tiras de papel cartolina. Realizaram-se seis diferentes experimentos ou situações relativas à: orientação de alvo, em relação ao plano do solo, (horizontal - $0^{\circ}$; inclinada - 45 ; vertical $\left.-90^{\circ}\right) \times$ vento $\left(0\right.$ e $\left.10 \mathrm{~km} \mathrm{~h}^{-1}\right)$. Para cada uma dessas situações, estudou-se o efeito de seis aplicações: três diferentes sentidos (cima para baixo, lateral e baixo para cima) x duas pontas de pulverização (SF 110-02 e LD 110-02). Avaliou-se o percentual de cobertura das aplicações nas duas faces dos alvos artificiais. Nesse sentido, o delineamento experimental foi em esquema fatorial (Sentido de aplicação x Ponta de pulverização x Face do alvo) para cada uma das seis situações de orientação de alvo e vento. Os resultados indicam que as faces opostas ao sentido da aplicação recebem níveis de cobertura menores em relação à face direcionada ao sentido da aplicação, e que nas faces direcionadas ao sentido da aplicação, alvos orientados de forma inclinada $\left(45^{\circ}\right)$ e vertical $\left(90^{\circ}\right)$, a ponta SF 110-02 promoveu maiores níveis de cobertura, independentemente da ação do vento.
\end{abstract}

Palavras-chave: tecnologia de aplicação, cobertura, alvo artificial, faces de alvo.

\begin{abstract}
Several factors influence the efficiency of the application: weather, equipment, chemical products, target and moment of application. Another aspect is the uniformity of the spray in different leave surfaces, because it influences in the absorption, translocation and control of the biological agents by contact products. The aim of this paper was to study the effect of different spray directions and nozzle in the percentage of coverage at artificial target (upper and lower faces) to six different situations about target orientation and wind. The artificial target was cardstock paper. It was realized six different experiments or situations: target orientation, in relation to the soil plan, (horizontal - $0^{\text {th }}$, inclined - $45^{\text {th }}$ and vertical $\left.-90^{\text {th }}\right) \times$ wind $\left(0\right.$ e $\left.10 \mathrm{~km} \mathrm{~h}^{-1}\right)$. For each situation, it was study six applications: three directions (top to bottom, side and bottom to up) x two nozzles (SF 110-02 e LD 110-02). It was evaluated the percentage of coverage in the two faces of the artificial target. Based on this, the experimental design was factorial (spray direction $x$ nozzle $x$ target face) to each situation of target orientation and wind. The results indicate that surfaces opposite to the direction spray receive lower levels of coverage in relation to the surfaces turned to the spray, and that in the surfaces turned to the spray on the inclined $\left(45^{\text {th }}\right)$ and vertical $\left(90^{\text {th }}\right)$ orientation the SF 110-02 nozzle promoted higher percentages of coverage, independently of the wind.
\end{abstract}

Key words: application technology, coverage, artificial target, target surfaces.

\section{INTRODUÇÃO}

A aplicação fitossanitária é uma das técnicas mais estudadas no âmbito agronômico, devido a sua

'Programa de Pós-graduação em Agronomia, Universidade Estadual de Londrina (UEL), 86047-000, Londrina, PR, Brasil. Email: gmigliorini@hotmail.com. Autor para correspondência.

"Departamento de Agronomia, Universidade Estadual de Maringá (UEM), Maringá, PR, Brasil.

IIIDepartamento de Agronomia, Universidade Estadual de Londrina (UEL), Londrina, PR, Brasil. 
inter-relação com as áreas de proteção de plantas (doenças, insetos e plantas daninhas) e ambiental (contaminação por deriva). Nesse sentido, os alvos artificiais são uma das ferramentas mais utilizadas em estudos ligados à tecnologia de aplicação, devido a sua fácil manipulação e sensibilidade nas diferentes formas de avaliações (cobertura e deposição) (BALAN et al., 2005; FIRVEDA et al., 2002).

A eficácia do controle químico depende da ação dos agentes químicos e fatores inerentes: ao momento biológico de controle, à cobertura e deposição do produto em diferentes extratos do dossel e faces foliares da cultura. Os produtos fitossanitários são aplicados em diversos alvos específicos e, para cada um desses, deve-se realizar aplicação com máxima precisão, aliado ao menor risco de contaminação do ambiente e aplicador (MATTHEWS, 2008). Com base nestes pressupostos, as plantas cultivadas são os principais alvos das aplicações, devendo, portanto, atentar para sua arquitetura e índice de área foliar (IAF) no momento da aplicação.

Elevados níveis de cobertura e penetração no dossel das plantas, em aplicações laterais (turboatomizadores) e de cima para baixo (pulverizadores de barra convencionais), podem ser obtidos pelo uso de maiores taxas de aplicação e gotas menores (FARINHA et al., 2009; VIANA et al., 2008). Porém, tais gotas são mais sujeitas à evaporação e desvio de sua trajetória quando da ação do vento (CUNHA, 2008; COSTA et al., 2007), reduzindo a cobertura esperada. Já em aplicações direcionadas de baixo para cima, gotas finas não necessariamente promovem maiores níveis de cobertura, uma vez que, nesse tipo de aplicação, as gotas têm seu deslocamento influenciado pelo movimento adquirido (momento linear), determinado pela velocidade e massa da gota pulverizada (ANTUNIASSI \& ABI SAAB, 1998).

Outro ponto que merece destaque refere-se à absorção dos produtos, intimamente ligado à espessura da cutícula. Estudando diferentes espécies de plantas, FERREIRA et al. (2002; 2003) evidenciaram que a espessura da cutícula varia entre as faces das folhas, apresentando menor espessura na face abaxial. Assim, as gotas pulverizadas devem alcançar tal face, a fim de aumentar a absorção e consequentemente a ação dos produtos aplicados.

O objetivo do trabalho foi estudar o efeito de diferentes sentidos de aplicação e pontas de pulverização no percentual de cobertura de alvos artificiais (face superior e inferior) para seis diferentes situações de orientação de alvo e vento.

\section{MATERIAL E MÉTODOS}

O experimento foi realizado na Universidade Estadual de Londrina sob condições de ambiente fechado para anular o efeito do vento externo. Condições de temperatura $\left(20^{\circ} \mathrm{C}\right)$ e umidade relativa do $\operatorname{ar}(70 \%)$ foram obtidas através de condicionador de ar, sendo estes monitorados pelo uso de termohigrômetro. Os alvos artificiais foram constituídos de tiras de papel cartolina branca de tamanho de $4 \mathrm{~cm} \times 10 \mathrm{~cm}$ (BALAN et al., 2005).

Realizaram-se seis diferentes experimentos - situações relativas à: orientação de alvo, em relação ao plano do solo, (horizontal $-0^{\circ}$; inclinada - $45^{\circ}$; vertical $\left.-90^{\circ}\right) \mathrm{x}$ vento $\left(0\right.$ e $\left.10 \mathrm{~km} \mathrm{~h}^{-1}\right)$, obtidas por meio de um ventilador com fluxo de ar constante no sentido perpendicular ao da aplicação. Para cada uma dessas situações, estudou-se o efeito de seis aplicações: três diferentes sentidos (cima para baixo, lateral e baixo para cima) x duas pontas de pulverização (SF 110-02 e LD 110-02). Avaliou-se o percentual de cobertura nas duas faces dos alvos artificiais. Nesse sentido, houve um delineamento experimental em esquema fatorial triplo, com seis repetições (10 tiras de papel cartolina cada), tendo como fatores sentido de aplicação, ponta de pulverização, face do alvo, para cada um das seis diferentes situações de orientação de alvo e vento.

A calda aplicada foi uma mistura de água + corante preto para tinta látex a $0,5 \%$. As pulverizações foram feitas através de um equipamento costal, equipado com válvula reguladora de pressão $(200 \mathrm{kPa})$, na vazão de $0,68 \mathrm{~L} \mathrm{~min}^{-1}$, velocidade de $6 \mathrm{~km} \mathrm{~h}^{-1}$ a uma distância de $0,50 \mathrm{~m}$ dos alvos. Segundo o fabricante, a pressão de trabalho utilizada promove distintos

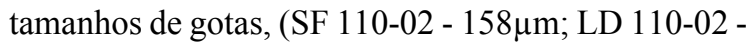
$205 \mu \mathrm{m})$. Após as aplicações, os alvos foram digitalizados em um Scanner com resolução de 300dpi, nas cores preta e branca. Essas cores foram transformadas pelo software Corel Draw 10 para as cores azul e amarelo (BALAN et al., 2005), sendo processadas posteriormente pelo software Conta-Gotas (CANTERI et al., 2001) para estimativa da porcentagem de cobertura das gotas no alvo. As análises estatísticas dos dados constituíram-se de análises de variâncias seguidas do teste Tukey a 5\% de probabilidade.

\section{RESULTADOS E DISCUSSÃO}

Analisando as aplicações sobre o alvo posicionado de forma horizontal, notam-se interações significativas entre: Sentido da aplicação x Face do alvo, quando na presença de vento; Sentido da aplicação x Ponta e Sentido da aplicação x Face, quando na ausência de vento (Tabela 1). Aplicações direcionadas à face do alvo, ou seja, de cima para baixo (face superior) e baixo para cima (face inferior), 
Tabela 1 - Porcentagens de cobertura no alvo posicionado horizontalmente para: Ponta de pulverização e interação entre Sentido da aplicação x Face, na presença de vento; e interações entre Sentido da aplicação x Ponta e Sentido da aplicação x Face, na ausência de vento.

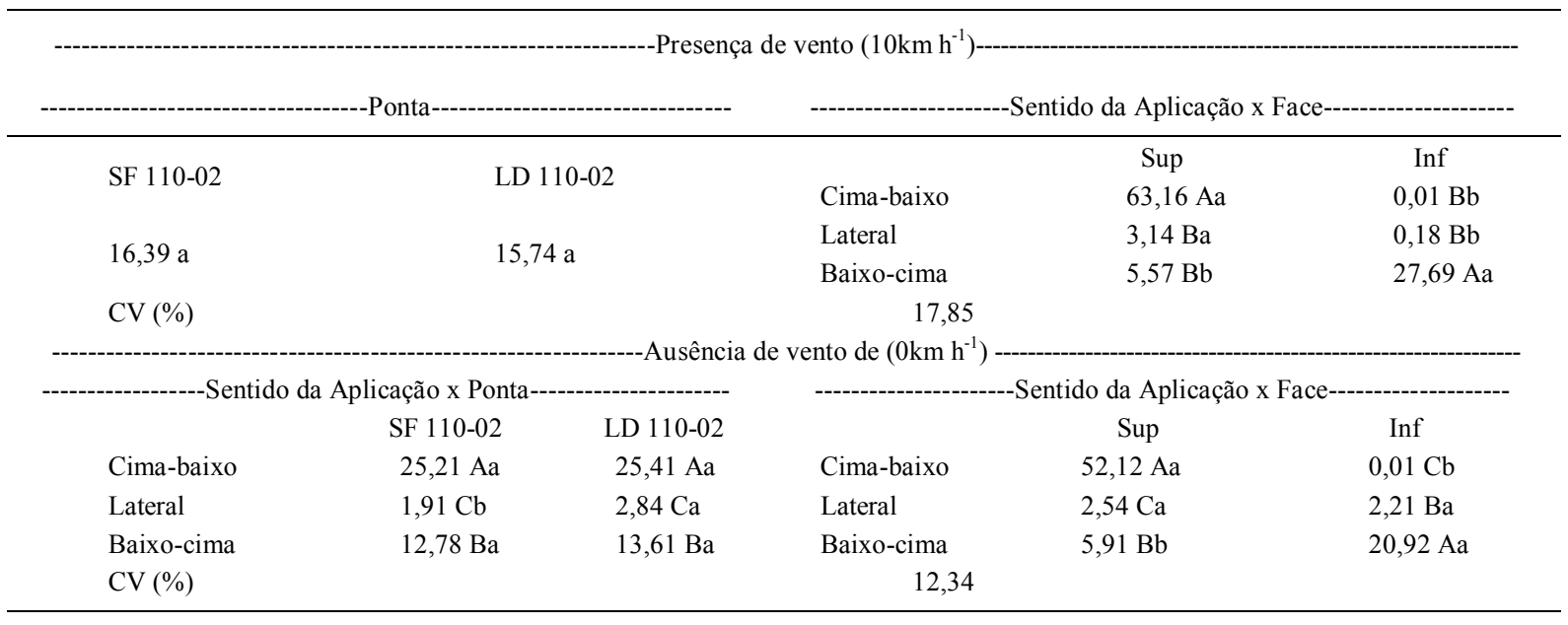

*Médias seguidas de mesma letra, maiúsculas nas colunas e minúsculas nas linhas, não diferem entre si pelo teste Tukey a 5\% de probabilidade. $* *$ Dados transformados para raiz quadrada de $\mathrm{x}+0,5$. Faces $(\operatorname{Sup}=$ Superior; Inf $=$ Inferior $)$.

promovem os maiores níveis de cobertura, independentemente da ponta de pulverização e do vento (Tabela 1). Dentre estas, pode-se observar que o sentido de cima para baixo apresenta maior nível de cobertura em relação ao sentido de baixo para cima, com valores superiores na ordem de 2 a 2,5 vezes.

Inexistem diferenças consideráveis entre as pontas de pulverização nas aplicações neste tipo de situação, salvo na aplicação lateral na ausência de vento, em que a ponta LD 110-02 proporcionou uma área coberta em torno de $2,84 \%$, significativamente superior a $1,91 \%$ da ponta $110 \mathrm{SF}-02$ (Tabela 1 ). Valores próximos de DMV proporcionados pelas pontas SF

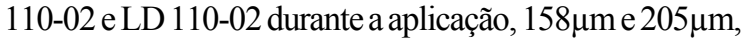
respectivamente, podem ser a razão pela qual não é possível verificar amplas diferenças significativas entre as pontas de pulverização com relação ao percentual de cobertura, haja vista que métodos de determinação da cobertura através do contraste gerado pela deposição das gotas podem ser de baixa sensibilidade em tamanhos de gotas muito próximos (FIRVEDA et al., 2002).

Quanto ao alvo posicionado de forma inclinada, verificam-se interações significativas triplas (Sentido da aplicação x Face do alvo x Ponta de pulverização) para ambas as situações de vento. As médias estão apresentadas em função das pontas de pulverização e faces dos alvos (Tabela 2). Novamente, as aplicações direcionadas às faces do alvo inclinado $\left(45^{\circ}\right)$, de cima para baixo e lateral (para a face superior) e de baixo para cima (para a face inferior), apresentaram maiores níveis de cobertura, independentemente da ação do vento (Tabela 2). Verifica-se ainda que a ponta de pulverização SF 110-02 promove maior porcentual de cobertura nas aplicações direcionadas às faces do alvo posicionado de forma inclinada, exceto na aplicação no sentido de baixo para cima, quando da ausência de vento. Gotas menores têm como característica a obtenção de maiores níveis de cobertura (FARINHA et al., 2009; VIANA et al., 2008 ), quando aplicadas de cima para baixo e/ou lateralmente, enquanto que gotas maiores podem promover percentuais superiores quando aplicadas de baixo para cima (sem o uso de jato transportado). Este fato é decorrente do movimento adquirido pelas gotas (momento linear), função da velocidade e da massa da gota, aumentando o deslocamento destas durante a aplicação de baixo para cima (ANTUNIASSI \& ABI SAAB, 1998).

Maiores níveis de cobertura proporcionados pelas gotas menores (SF 110-02) na posição inclinada, não verificado na posição horizontal, podem estar ligados a menor área projetada da face do alvo em relação aos sentidos da aplicação aqui estudados, sugerindo que a área do alvo artificial, em experimentos ligados à tecnologia de aplicação, influencia nos resultados de cobertura.

No alvo posicionado verticalmente, verificam-se interações significativas entre os três fatores estudados (Sentido da aplicação x Face do alvo x Ponta de pulverização) em ambas as situações de vento. As médias estão apresentadas em função das 
Tabela 2 - Porcentagens de cobertura no alvo posicionado de forma inclinada para interações triplas (Sentido da aplicação x Face x Ponta, na presença $\left(10 \mathrm{~km} \mathrm{~h}^{-1}\right)$ e ausência $\left(0 \mathrm{~km} \mathrm{~h}^{-1}\right)$ de vento.

\begin{tabular}{|c|c|c|c|c|c|c|c|}
\hline \multicolumn{4}{|c|}{ 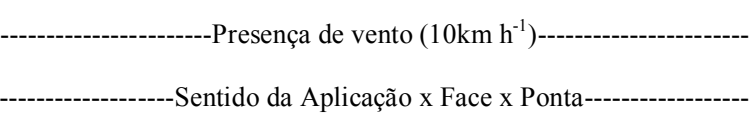 } & \multicolumn{4}{|c|}{ 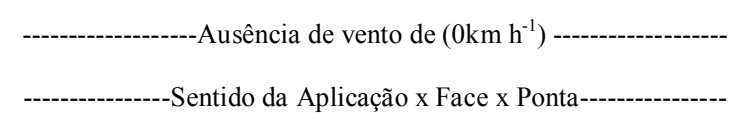 } \\
\hline Sentido da Aplicação & Face & SF110-02 & LD110-02 & Sentido da Aplicação & Face & SF110-02 & LD110-02 \\
\hline Cima-baixo & Sup & $57,22 \mathrm{Aa}$ & $48,06 \mathrm{Ab}$ & Cima-baixo & Sup & $42,1 \mathrm{Aa}$ & $33,17 \mathrm{Ab}$ \\
\hline Cima-baixo & $\operatorname{Inf}$ & $0,01 \mathrm{Ba}$ & $0,01 \mathrm{Ba}$ & Cima-baixo & Inf & $0,01 \mathrm{Ba}$ & $0,01 \mathrm{Ba}$ \\
\hline Lateral & Sup & $63,88 \mathrm{Aa}$ & $52,01 \mathrm{Ab}$ & Lateral & Sup & $47,56 \mathrm{Aa}$ & $41,89 \mathrm{Ab}$ \\
\hline Lateral & $\operatorname{Inf}$ & $0,01 \mathrm{Ba}$ & $0,01 \mathrm{Ba}$ & Lateral & $\operatorname{Inf}$ & $0,00 \mathrm{Ba}$ & $0,01 \mathrm{Ba}$ \\
\hline Baixo-cima & Sup & 7,09 Ba & $4,89 \mathrm{Ba}$ & Baixo-cima & Sup & $3,72 \mathrm{Ba}$ & $4,02 \mathrm{Ba}$ \\
\hline Baixo-cima & $\operatorname{Inf}$ & $22,53 \mathrm{Aa}$ & $16,06 \mathrm{Ab}$ & Baixo-cima & $\operatorname{Inf}$ & $19,56 \mathrm{Aa}$ & $19,92 \mathrm{Aa}$ \\
\hline CV (\%) & & 14,19 & & CV (\%) & & 11,01 & \\
\hline
\end{tabular}

*Médias seguidas de mesma letra, maiúsculas nas colunas e minúsculas nas linhas, não diferem entre si pelo teste Tukey a 5\% de probabilidade. **Letras minúsculas referem-se à comparação de pontas de pulverização, e maiúsculas à comparação de faces (Sup $=$ Superior; Inf = Inferior) para um único sentido de aplicação. ***Dados transformados para raiz quadrada de $\mathrm{x}+0,5$.

pontas de pulverização e faces do alvo (Tabela 3). Resultados referentes aos alvos posicionados de forma vertical $\left(0^{\circ}\right)$ evidenciam que: a face inferior do alvo apresenta percentual de cobertura ínfima em relação à face superior, independentemente do sentido da aplicação; e que a aplicação direcionada, neste caso aplicação lateral voltada à face superior, independentemente da ação do vento, apresenta um nível de cobertura substancialmente superior $(>60 \%)$ em relação aos demais sentidos de aplicação $(<10 \%)$ (Tabela 3).

Nota-se ainda, na tabela 3 , que a ponta SF 110-02 apresenta níveis de cobertura superiores somente na face superior do alvo, sobretudo nas aplicações laterais (independentemente do vento), aplicações de baixo para cima (na presença de vento) e de cima para baixo (na ausência de vento). Para a face superior na aplicação lateral, o menor tamanho de gotas gerado pela ponta SF 110-02 resulta em maiores níveis de cobertura, pois tal face está voltada para o mesmo sentido da aplicação. Já para os demais sentidos de aplicação, os resultados não são passíveis de inferências plausíveis, devido ao baixo nível de cobertura verificado.

Os resultados deste trabalho são notórios quanto às evidências de que os maiores níveis de cobertura foram alcançados somente nas aplicações direcionadas à face do alvo, independentemente do

Tabela 3 - Porcentagens de cobertura no alvo posicionado verticalmente para: interações entre Sentido da aplicação x Face x Ponta, na presença $\left(10 \mathrm{~km} \mathrm{~h}^{-1}\right)$ e ausência $\left(0 \mathrm{~km} \mathrm{~h}^{-1}\right)$ de vento.

\begin{tabular}{|c|c|c|c|c|c|c|c|}
\hline Sentido da Aplicação & Face & SF110-02 & LD110-02 & Sentido da Aplicação & Face & SF110-02 & LD110-02 \\
\hline Cima-baixo & Sup & 7,74 Aa & $8,41 \mathrm{Aa}$ & Cima-baixo & Sup & $11,87 \mathrm{Aa}$ & $5,43 \mathrm{Ab}$ \\
\hline Cima-baixo & Inf & $0,57 \mathrm{Ba}$ & $2,15 \mathrm{Ba}$ & Cima -baixo & $\operatorname{Inf}$ & $0,00 \mathrm{Ba}$ & $0,05 \mathrm{Ba}$ \\
\hline Lateral & Sup & $82,68 \mathrm{Aa}$ & $64,39 \mathrm{Ab}$ & Lateral & Sup & $63,32 \mathrm{Aa}$ & $54,58 \mathrm{Ab}$ \\
\hline Lateral & $\operatorname{Inf}$ & $0,00 \mathrm{Ba}$ & $0,01 \mathrm{Ba}$ & Lateral & $\operatorname{Inf}$ & $0,01 \mathrm{Ba}$ & $0,03 \mathrm{Ba}$ \\
\hline Baixo-cima & Sup & 7,67 Aa & $4,63 \mathrm{Ab}$ & Baixo-cima & Sup & $0,01 \mathrm{Aa}$ & $0,01 \mathrm{Ba}$ \\
\hline Baixo-cima & $\operatorname{Inf}$ & $0,90 \mathrm{Ba}$ & $0,94 \mathrm{Ba}$ & Baixo-cima & Inf & $2,42 \mathrm{Ba}$ & $2,48 \mathrm{Aa}$ \\
\hline CV $(\%)$ & & 20,72 & & CV $(\%)$ & & 20,51 & \\
\hline
\end{tabular}

*Médias seguidas de mesma letra, maiúsculas nas colunas e minúsculas nas linhas, não diferem entre si pelo teste Tukey a $5 \%$ de probabilidade. **Letras minúsculas referem-se à comparação de pontas de pulverização, e maiúsculas à comparação de faces (Sup = Superior; Inf $=$ Inferior) para um único sentido de aplicação. $* * *$ Dados transformados para raiz quadrada de $\mathrm{x}+0,5$.

Ciência Rural, v.42, n.4, abr, 2012. 
vento $\left(0 \mathrm{e} 10 \mathrm{~km} \mathrm{~h}^{-1}\right)$. Em alvos artificiais, BALAN et al. (2005) encontraram níveis de cobertura ínfimos na face oposta ao sentido da aplicação. No tocante à deposição, resultados semelhantes foram encontrados por MASKI \& DURAIRAJ (2010), alcançando maiores depósitos na face contrária, somente com o uso de eletrificação de gotas. Outros autores, estudando depósitos nas diferentes faces das folhas de soja (CHRISTOVAM et al., 2010a, b), encontraram menores valores nestas mesmas faces abaxiais, com redução gradativa ao longo dos extratos do dossel, sendo parte destes depósitos decorrentes de diferentes angulações e sobreposições foliares da cultura no campo.

A maioria das aplicações, por área cultivada, é efetuada de cima para baixo (equipamentos de barras) e lateralmente (turbo-atomizadores), ou seja, aplicações direcionadas à face adaxial das folhas. Nesse sentido, os resultados dão a conotação de que níveis de cobertura reduzidos atingem a face inferior das folhas, o que resultaria na redução do controle de pragas que habitam as faces abaxiais, por exemplo, a mosca branca (Bemisia tabaci) no feijoeiro (RAETANO \& BAUER, 2004), como também em aplicações de fungicidas de contato que requerem uma cobertura uniforme no dossel da cultura (PERGHER et al., 1997). A absorção dos produtos sistêmicos também está ligada a esse contexto, uma vez que a espessura da cutícula é relativamente menor na face abaxial das folhas (FERREIRA et al., 2002; 2003), o que aumenta a absorção destes nesta face (WANG \& LIU, 2007).

Equipamentos com assistência de ar (MATTHEWS, 2008) e eletrostáticos (SERRA et Al., 2008) possibilitam maior cobertura e penetração das gotas no dossel, mesmo nas faces abaxiais, devido à movimentação do dossel da cultura e atração das gotas para folhas, respectivamente. Entretanto, outras tecnologias podem ser utilizadas: associação de pingentes em equipamentos de barra convencional aplicações de cima para baixo + baixo para cima (PEIXOTO et al., 2009); tecnologia canopy opener (abridores de dossel), utilizadas em pulverizadores de barra convencional (ZHU et al., 2008).

Por fim, o entendimento acerca dos fatores inerentes à aplicação, para as mais diversas situações, é de indubitável importância, por assegurar que estas se tornem mais eficazes e causem menores influências no meio ambiente futuramente.

\section{CONCLUSÃO}

Pode-se concluir que as faces opostas aos sentidos de aplicação recebem níveis de cobertura reduzidos e que, nas aplicações direcionadas à face superior dos alvos, nas orientações inclinada e vertical, a ponta SF 110-02 promoveu maiores níveis de cobertura, independentemente da ação do vento.

\section{REFERÊNCIAS}

ANTUNIASSI, U.R.; ABI SAAB, O.J.G. Avaliação da porcentagem de cobertura pelas gotas de aplicações fitossanitárias em videiras. Revista Brasileira de Engenharia Agrícola e Ambiental, v.2, n.2, p.205-211, 1998. Disponível em: <http:/ /www.agriambi.com.br/revista/v2n2/205.pdf $>$. Acesso em: 02 out. 2011 .

BALAN, M.G. et al. Pulverização em alvos artificiais: avaliação com o software conta-gotas. Ciência Rural, v.35, n.4, p.916919, 2005. Disponível em: <http://www.scielo.br/ s cielo.php? s cript $=$ sci_arttext \& pid = S $0103-$ $84782005000400026 \& \operatorname{lng}=\mathrm{en} \& \mathrm{nrm}=\mathrm{iso}>$. Acesso em: 06 out. 2011. doi: 10.1590/S0103-84782005000400026.

CANTERI, M.G. et al. Conta-gotas: sistema para análise de eficiência de pulverização. In: CONGRESSO PAULISTA DE FITOPATOLOGIA, 24., 2001, Piracicaba, SP. Summa Phytopathologica. Jaboticabal: Grupo Paulista de Fitopatologia, 2001. V.27, p.136.

CHISTOVAM, R.S. et al. Air-assistance and low volume application control of Asian rust soybean crop. Journal of Plant Protection Research, v.50, n.3, p.354-359, 2010. Disponível em: <http://versita.metapress.com/content/ x017166217r25g7u/fulltext.pdf>. Acesso em: 02 out. 2011. doi: $10.2478 / \mathrm{v} 10045-010-0060-y$.

CRHISTOVAM, R.S. et al. Assistência de ar na barra de pulverização no controle da ferrugem asiática da soja. Bragantia, v.69, n.1, p.231-238, 2010. Disponível em: <http:/ /www.scielo.br/scielo.php?script $=$ sci arttext\&pid $=$ S0006$87052010000100029 \& \operatorname{lng}=\mathrm{en} \& \mathrm{nrm}=\mathrm{iso}>$. Acesso em: 03 out. 2011. doi: 10.1590/S0006-87052010000100029.

COSTA, A.G.F. et al. Efeito da intensidade do vento, da pressão e de pontas de pulverização na deriva de aplicações de herbicidas em pré-emergência. Planta Daninha, v.25, n.1, p.203-210, 2007. Disponível em: <http://www.scielo.br/ scie lo.php? script $=$ sci_arttext \& pid $=$ S $0006-$ $87052010000100029 \& \operatorname{lng}=\mathrm{en} \& \mathrm{nrm}=\mathrm{iso}>$. Acesso em: 03 out. 2011. doi: 10.1590/S0006-87052010000100029.

CUNHA, J.P.A.R. Simulação da deriva de agrotóxicos em diferentes métodos de aplicação. Revista Ciência Agronômica, v.39, n.4, p.487-493, 2008. Disponível em: $<$ http://www.ccarevista.ufc.br/seer/index.php/ccarevista/article/ viewFile/378/274.>. Acesso em: 03 out. 2011

FARINHA, J.V. et al. Deposição da calda de pulverização em cultivares de soja no estádio R1. Ciência Rural, v.39, n.6, p.1738-1744, 2009. Disponível em: <http://www.scielo.br/ scie lo.php? s cript=sci_arttext \& pid = S $0103-$ $84782009000600016 \& \operatorname{lng}=$ pt\&nrm=iso $>$. Acesso em: 06 out. 2011. doi: 10.1590/S0103-84782009000600016.

FERREIRA, E.A. Estudos anatômicos de folhas de plantas daninhas. I - Nicandra physaloides, Solanum viarum, Solanum americanum e Raphanus raphanistrum. Planta Daninha, v.20, n.2, p.159-167, 2002. Disponível em: <http:// 
www.scielo.br/scielo.php?script $=$ sci_arttext\&pid $=\mathrm{S} 0100-$ $83582002000200001 \& \operatorname{lng}=\mathrm{en} \& \mathrm{nrm}=\mathrm{iso}>$. Acesso em: 06 out. 2011. doi: 10.1590/S0100-83582002000200001.

FERREIRA, E.A. Estudos anatômicos de folhas de espécies de plantas daninhas de grande ocorrência no Brasil. IV - Amaranthus deflexus, Amaranthus spinosus, Alternanthera tenella $e$ Euphorbia heterophyll. Planta Daninha, v.21, n.2, p.263271, 2003. Disponível em: <http://www.scielo.br/ s cielo.php? script=sci_art text \& pid = S $0100-$ $83582003000200012 \& \operatorname{lng}=\mathrm{en} \& \mathrm{nrm}=\mathrm{iso}>$. Acesso em: 06 out. 2011. doi:10.1590/S0100-83582003000200012.

FIRVEDA, M.C. et al. Uso de software para analise de imagem em avaliação de cobertura de pulverização. Bragantia, v.61, n.3, p.305-310, 2002. Disponível em: <http://www.scielo.br/ scie 1 o.php? script=sci_art text \& pid = S $0006-$ $87052002000300013 \& \operatorname{lng}=\mathrm{en} \& \mathrm{nrm}=\mathrm{iso}>$. Acesso em: 02 out. 2011. doi: 10.1590/S0006-87052002000300013.

MASKI, D.; DURAIRAJ, D. Effects of charging voltage, application speed, target height, and orientation upon charged spray deposition on leaf abaxial and adaxial surfaces. Crop Protection, v.29, p.134-141, 2010. Disponível em: <http:// www.sciencedirect.com/science/journal/02612194/29/2>. Acesso em: 06 out. 2011. doi: 10.1016/j.cropro.2009.10.006.

MATTHEWS, G.A. Developments in application technology. Environmentalist, v.28, p.19-24, 2008. Disponível em: $<$ http://www.springerlink.com/content/b66hh751t1xw7214/ export-citation>. Acesso em: 06 out. 2011. doi:10.1007/ s10669-007-9039-2.

PEIXOTO, M.F. et al. Controle e perdas provocadas por Mahanarva fimbriolata (Stal) (Hemiptera: Cercopidae) em cana de açúcar. Global Science and Technology, v.2, n.1, p.114122, 2009. Disponível em: <http://www.cefetrv.edu.br/ periodicos/index.php/gst/article/view/41/14>. Acesso em: 02 out. 2011.

PERGHER, G. et al. Foliar deposition and pesticide losses from three air-assisted sprayers in a hedgerow vineyard. Crop
Protection, v.16, n.1, p.25-33, 1997. Disponível em: <http:/ / w w w.sciencedirect.com/s cience/article/pi i / S0261219496000543>. Acesso em: 02 out. 2011. doi:10.1016/ S0261-2194(96)00054-3.

RAETANO, C.G.; BAUER, F.C. Deposição e perdas da calda em feijoeiro em aplicação com assistência de ar na barra pulverizadora. Bragantia, v.63, n.2, p.309-315, 2004. Disponível em: <http:/ /www.scielo.br/scielo.php?script $=$ sci_arttext\&pid $=\mathrm{S} 0006$ $87052004000200016 \& \operatorname{lng}=$ en $\& n r m=$ iso $>$. Acesso em: 02 out. 2011. doi:10.1590/S0006-87052004000200016.

SERRA, M.E. et al. Pontas de pulverização e eletrificação das gotas na deposição da calda em plantas de crisântemo. Pesquisa Agropecuária Brasileira, v.43, n.4, p.479-485, 2008. Disponível em: <http://www.scielo.br/ scielo.php? script=sci_art text \& pid=S $0100-$ 204X2008000400006\&lng=en $\& n r m=i s o>$. Acesso em: 06 out. 2011. doi:10.1590/S0100-204X2008000400006.

VIANA, R.G. et al. Deposição de gotas no dossel da soja por diferentes pontas de pulverização hidráulica e pressões de trabalho. Engenharia na Agricultura, v.16, n.4, p.428435, 2008. Disponível em: <http://www.seer.ufv.br/seer/ index.php/reveng/article/viewFile/59/31>. Acesso em: 06 out. 2011

WANG, C.J.; LIU, Z.Q. Foliar uptake of pesticides - Present status and future challenge. Pesticide Biochemistry and Physiology, v.87, n.1, p.1-8, 2007. Disponível em: <http:// ww w.sciencedirect.com/science/article/pi i/ S0048357506000691>. Acesso em: 06 out. 2011.

ZHU, H. et al. Development of a canopy opener to improve spray deposition and coverage inside soybean canopies: part 1 . Mathematical models to assist opener development. Transactions of ASABE-American Society of Agricultural and Biological engineers, v.51, n.6, p.1905-1912, 2008. Disponível em: <http://asae.frymulti.com/ abstract.asp?aid=25389\&t=2.>. Acesso em: 06 out. 2011. 